\title{
Acute-on-chronic slipped femoral epiphysis following posterior spinal fusion due to idiopathic scoliosis: Case report
}

\author{
ELENI PAPPA ${ }^{1}$, FOTIOS KAKRIDONIS ${ }^{1}$, IOANNIS A. TRANTOS ${ }^{2}$, PAVLOS KATSOULIS ${ }^{2}$, \\ IOANNIS CHATZIKOMNINOS $^{1}$ and JOHNANASTASOPOULOS ${ }^{2}$ \\ ${ }^{1}$ Department of Spine Surgery and Scoliosis, KAT General Hospital, 14561 Athens; \\ ${ }^{2}$ Second Orthopaedic Department of General Children Hospital 'Agia Sofia', 11527 Athens, Greece
}

Received December 5, 2021; Accepted January 10, 2022

DOI: $10.3892 / b r .2022 .1505$

\begin{abstract}
The aim of this case report is to present a rare case of acute slipped femoral capital on a chronic slipped capital femoral epiphysis (SCFE) after spinal fusion due to idiopathic scoliosis. A 14 year old male patient underwent posterior spinal fusion due to idiopathic thoracic scoliosis. Post-operatively, the patient presented with acute pain in the left hip and a reduced range of motion, which revealed acute SCFE. The patient was then referred to the Second Orthopaedic Department of Agia Sofia Children Hospital in Athens, and underwent percutaneous pinning of the left femur, after which he was discharged uneventfully. The follow up was excellent with no impact on the patient's daily life. The case described is extremely rare in the current literature. The significance of the pre-operative planning is underlined by this case, as well as the need for the spinal surgeon to be aware of the possibility of acute pain in the hip in young adolescents, as SCFE is more common amongst this demographic.
\end{abstract}

\section{Introduction}

Slipped capital femoral epiphysis (SCFE) is a relatively common adolescent hip disorder, accounting for $\sim 10.8$ cases per 100,000 patients (1). This is explained by the fact that the physis is exhibits prominent levels of lysis during growth. The risk of epiphyseal displacement is also affected by normal proximal femoral development, physeal orientation, acetabular morphology as well as the endocrinological status of the adolescent $(1,2)$. During SCFE, a mechanical overload to the proximal femoral physis occurs, which leads to an anterior translation and external rotation of the metaphysic, respecting the upper femoral epiphysis $(3,4)$. Regarding SCFE symptomatology, limping and pain in the affected groin, lateral or

Correspondence to: $\mathrm{Dr}$ Eleni Pappa, Department of Spine Surgery and Scoliosis, KAT General Hospital, Nikis 2 Street, 14561 Athens, Greece

E-mail: helenapp27@yahoo.gr

Key words: slipped femoral epiphysis, idiopathic scoliosis posterior hip, thigh or ipsilateral knee are commonly observed. SCFE is also likely to present as knee pain, which may account for the high rates of misdiagnoses of this condition (5).

Several classification systems have been developed for SCFE. According to the duration of the symptoms, SCFE can be classified as acute, chronic or acute-on-chronic (6). Acute SCFE presents within 3 weeks of the onset of symptoms, and accounts for $10-15 \%$ of all SCFE cases. Moreover, acute SCFE is associated with a higher rate of avascular necrosis and can occur after trauma or an identifiable inciting injury (6). Chronic SCFE, accounts for $\sim 85 \%$ of cases, and implies at least 3 weeks of symptoms. Patients with predisposing symptoms of pain and limping, who then experience a new injury exhibit immediate worsening, the so-called acute-on-chronic slip (7). Loder et al (8) introduced the concept of physeal stability, categorizing SCFE as either stable or unstable based on the subject's ability to bear weight.

A patient with unstable SCFE experiences severe hip pain that does not allow for a normal gait (8). The patient will often have a medical history involving the hip, thigh and/or knee pain, and a previous trauma (usually minor and does not justify the condition alone). An affected patient tends to externally rotate the affected hip and counteracts any passive movement of the hip when lying down. The rate of osteonecrosis in unstable SCFE is increased to $24 \%$ (9). Additional grading is based on the radiographic severity, which is described by epiphyseal displacement as a fraction of the total physeal diameter (10). Using this grading, slips can be mild $(<33 \%)$, moderate $(33-50 \%)$ or severe $(>50 \%)$. One of the most commonly used classifications is the slip angle of Southwick (11), in which the difference in the angle subtended by the proximal femoral physis and the ipsilateral femoral shaft are compared between affected and unaffected sides (12). SCFE requires surgical management, except in rare instances, as stabilization of the epiphysis and early fusion of the proximal femoral physis prevents further displacement.

Regarding scoliosis, it is defined as a lateral curvature of the spine $>10^{\circ}$ on a radiograph, which is typically associated with spinal rotation. The three types of scoliosis are congenital, idiopathic and neuromuscular. Idiopathic scoliosis specifically is classified into infantile idiopathic scoliosis, which is identified in patients <3 years old; juvenile idiopathic scoliosis, which appears in children aged 3-10 years, and adolescent 
scoliosis in patients $>10$ years (13). Dickson and Archer (14) proposed a two-group classification system that included early onset ( $\leq 5$ years old) and late onset ( $\geq 6$ years old) scoliosis, since juvenile scoliosis is rare $(15,16)$ The prevalence of curves $>30^{\circ}$ is $\sim 0.2 \%$, and it is $<0.1 \%$ for curves $\geq 40^{\circ}$ (17).

The main cause of Adolescent Idiopathic Scoliosis has not been precisely identified, and it may be multifactorial. Genetics are hypothesized to play a leading role in the occurrence of scoliosis; however no mode of inheritance has been identified as of yet, to the best of our knowledge. Skeletally immature patients have a risk of curvature progression of $\sim 20 \%$ for curvatures of $20^{\circ}$, a risk of $60 \%$ at $30^{\circ}$ and $90 \%$ at $50^{\circ}(15)$. More severe curves are often associated with physical problems, such as increased back pain, cardiorespiratory dysfunction proportional to the severity of the curve, and an increased risk of pulmonary heart disease and death with curves $>100^{\circ}$ (18). Additionally, the psychological distress is also elevated with greater curvatures.

The aim of this case report is to present a rare case of acute-on-chronic SCFE in a patient undergoing surgical fixation of idiopathic scoliosis.

\section{Case report}

A male patient aged 14 with a health record of supraventricular tachycardia under medication for the past 2 years, BMI $=30 \mathrm{~kg} / \mathrm{m}^{2}$ and chronic SCFE of the left hip, was admitted to the Department of Spine Surgery and Scoliosis at KAT General Hospital, for surgical fixation of idiopathic scoliosis of the thoracic spine, with a Cobb angle of $45^{\circ}$. Left hip SCFE was irrelevant to the existing scoliosis and curvature, as there are no case series available in the literature describing any relevance between the scoliosis curvature and the hip SCFE, to the best of our knowledge.

A posterior lumbar fusion with instrumentation from T3 to L3 was performed under general anesthesia; however, post-operatively the patient reported acute pain in the left hip joint. Emergent postoperative imaging revealed an acute SCFE on the grounds of the chronic SCFE on the left hip. However there were no available pre-operative pelvic radiographs in the patient's history regarding an existing SCFE of the left hip. The patient was immediately referred to the Second Department of Orthopaedics at the 'Agia Sofia' Children's Hospital of Athens to undergo surgical intervention for the SCFE. The patient underwent percutaneous pin fixation of the left hip with a single screw, and discharged without any events. During a follow-up on 1 month after the operation, the patient showed both clinical and radiological recovery. On the last follow-up, 3 years after the operation, the patient was satisfied with his recovery, with a VAS score of $98 / 100$, and he reported a full return to his daily activities. The patient provided informed consent for publication of this case report (Figs. 1 and 2).

\section{Discussion}

Reviews of large national databases in the USA report an SCFE incidence rate of 10,117 per 100,000, with a 1.4:2.0 female/male ratio (1). The average age of diagnosis is 12 years, and the majority of patients who present with SCFE who are outside the age range of 10-16 represent atypical SCFE,
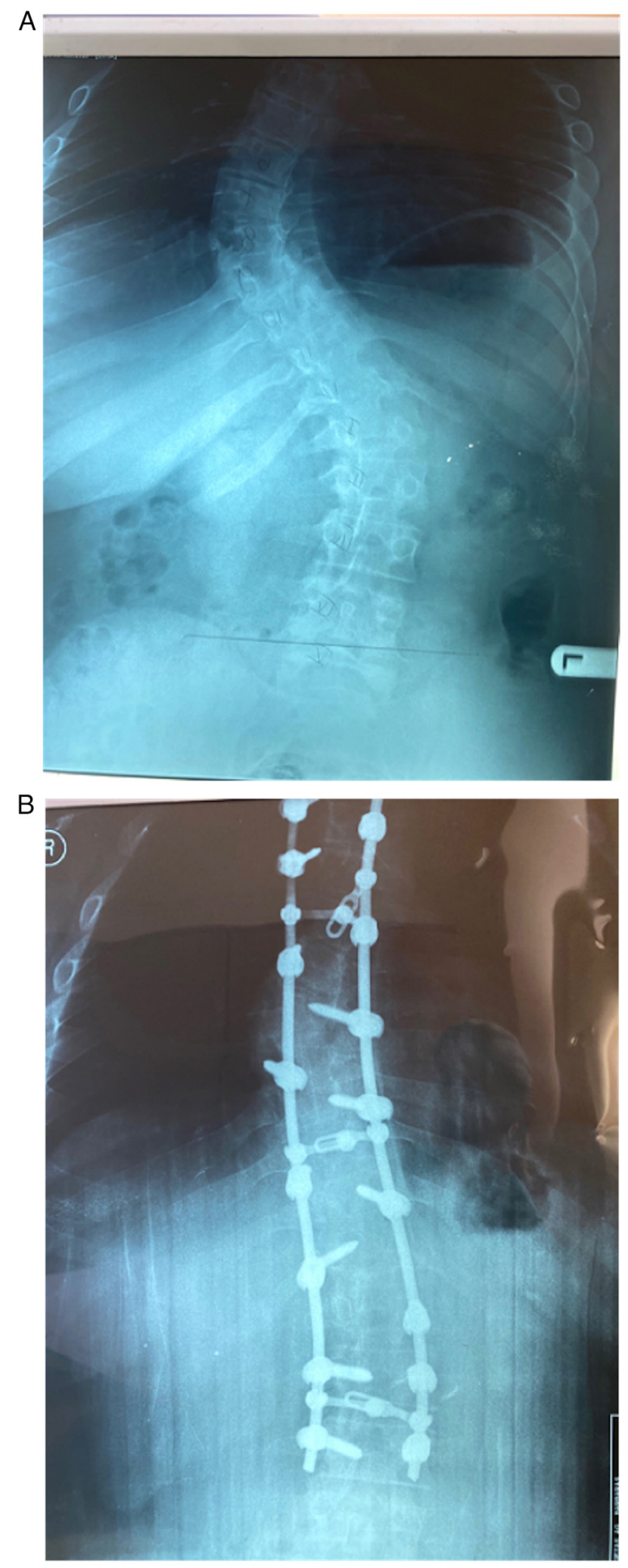

Figure 1. Radiographs of the thoracolumbar spine. (A) Pre-operative and (B) post-operative radiographs of the thoracolumbar spine after spinal fusion for idiopathic scoliosis (Cobb angle $45^{\circ}$ ).

possibly associated with endocrinological diagnoses (2). Although a common presentation of SCFE is that of an obese, hypogonadal male during an adolescent growth spurt, most SCFEs occur in the absence of an endocrine disorder (3). Conversely, the goal of scoliosis management is to prevent 

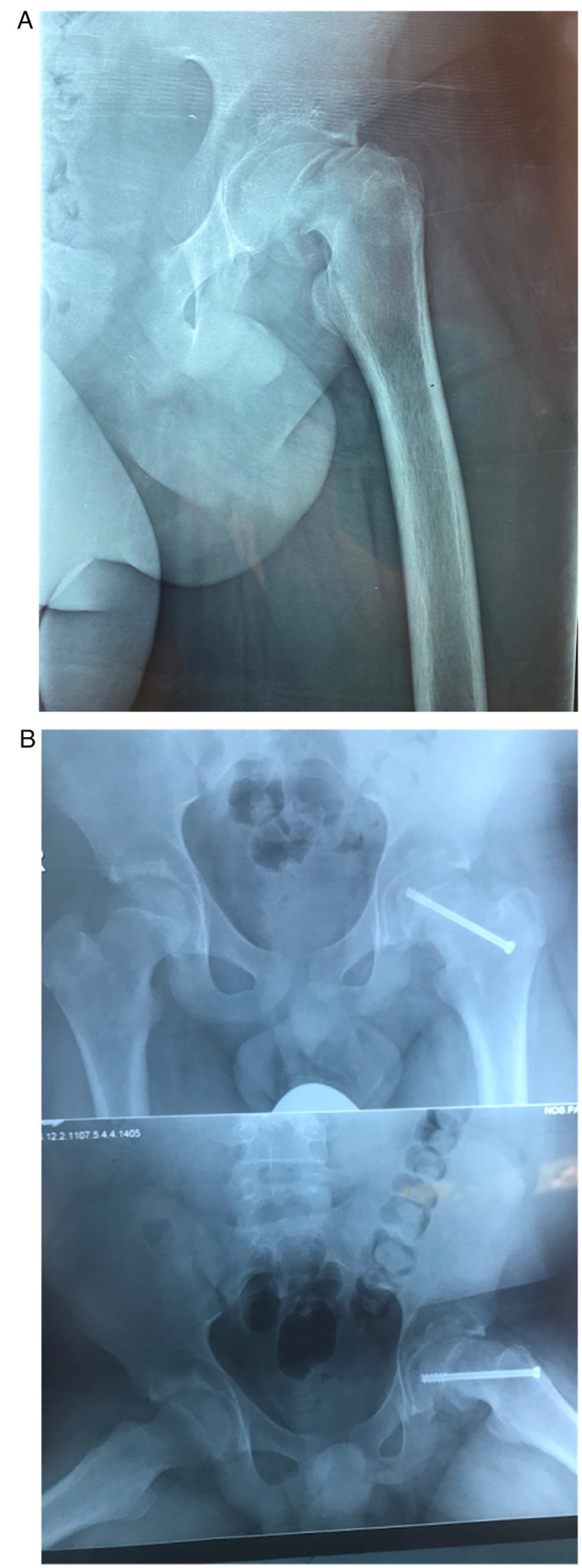

Figure 2. Radiographs of the femoral pinning. (A) Pre-operative and (B) post-operative radiographs of the femoral pinning due to acute slipped capital femoral epiphysis.

further progression of the curvature. Management decisions are made based on the curve severity at presentation, pattern and location of curvature, and growth potential of the patient (chronological age, menarche status and Risser sign) (13).
The majority of adolescents will not require intervention and $<10 \%$ require active treatment (17). Management options include observation and nonsurgical or surgical treatment. The goals of surgical treatment are to prevent progression, achieve maximal permanent correction of deformity, improve appearance and keep short-term and long-term complications to a minimum. Surgery is typically performed in patients with Cobb angles $>45^{\circ}$; however, additional factors, including age, curve progression and symptoms, such as pulmonary compromise, are important factors to be considered $(19,20)$.

The presence of an acute slipped femoral capital on an existing chronic SCFE simultaneously with surgical fixation of idiopathic scoliosis has not yet described in the existing literature, to the best of our knowledge. Of particular interest in this case is the cause of the acute slippage of the femoral capital right post-operatively. The patients position during the surgical instrumentation of the spine, which is a prone position under general anesthesia in four post frame with both hips at $90^{\circ}$ flexion, could be a reason that is likely to have led to this event. Additionally, the metabolic status of the patient, which included obesity, chronic asthma and cardiac tachyarrhythmias under ablation and constant medication, may have also contributed to the factors that led to a slipped femoral capital from the beginning. Prevention of the progress of any existing SCFE in patients undergoing spinal surgery is essential, including thorough radiographic pre-operative control and analysis of their detailed medical history.

In conclusion, the current case report, which is extremely rare in the current literature, describes the significance of pre-operative planning for each procedure in pediatric orthopedic surgery, particularly in patients with a medical history. The spinal surgeon should consider, in every acute change, the clinical presentation of the patient, especially in the hip after surgical interventions of the spine in pediatric patients, as $\mathrm{SCFE}$ is quite common during early adolescence.

\section{Acknowledgements}

Not applicable.

\section{Funding}

No funding was received.

\section{Availability of data and materials}

The datasets used and/or analyzed during the present study are available from the corresponding author on reasonable request.

\section{Authors' contributions}

EP, FK, IAT and PK collected the patients' medical information and scientific data, analyzed them and wrote the manuscript. IC and JA conceived the study, interpreted the data and edited the manuscript.. All authors read and approved the final manuscript.

\section{Ethics approval and consent to participate}

Not applicable. 


\section{Patient consent for publication}

The patient consented for publication of their data.

\section{Competing interests}

The authors declare that they have no competing interests.

\section{References}

1. Georgiadis A and Zaltz I: Slipped capital femoral epiphysis: How to evaluate with a review and update of treatment. Pediatr Clin North Am 61: 1119-1135, 2014.

2. Loder RT, Starnes T and Dikos G: Atypical and typical (idiopathic) slipped capital femoral epiphysis. Reconfirmation of the age-weight test and description of the height and age-height tests. J Bone Joint Surg Am 88: 1574-1581, 2006.

3. Mann DC, Weddington J and Richton S: Hormonal studies in patients with slipped capital femoral epiphysis without evidence of endocrinopathy. J Pediatr Orthop 8: 543-545, 1988.

4. Kocher MS, Bishop JA, Weed B, Hresko MT, Millis MB, Kim YJ and Kasser JR: Delay in diagnosis of slipped capital femoral epiphysis. Pediatrics 113: e322-e325, 2004.

5. Kaplan SR and Klinghoffer L: Knee pain in slipped femoral capital epiphysis causing a delay in diagnosis. Am J Surg 101: 798-802, 1961.

6. Aronsson DD and Loder RT: Treatment of the unstable (acute) slipped capital femoral epiphysis. Clin Orthop Relat Res: 99-110, 1996.

7. Aadalen RJ, Weiner DS, Hoyt W and Herndon CH: Acute slipped capital femoral epiphysis. J Bone Joint Surg Am 56: 1473-1487, 1974.

8. Loder RT, Richards BS, Shapiro PS, Reznick LR and Aronson DD: Acute slipped capital femoral epiphysis: The importance of physeal stability. J Bone Joint Surg Am 75 1134-1140, 1993.
9. Zaltz I, Baca G and Clohisy JC: Unstable SCFE: Review of treatment modalities and prevalence of osteonecrosis. Clin Orthop Relat Res 471: 2192-2198, 2013.

10. Wilson PD, Jacobs B and Schecter L: Slipped capital femoral epiphysis: An end-result study. J Bone Joint Surg Am 47: 1128-1145, 1965.

11. Southwick WO: Osteotomy through the lesser trochanter for slipped capital femoral epiphysis. J Bone Joint Surg Am 49: 807-835, 1967.

12. Loder RT: Effect of femur position on the angular measurement of slipped capital femoral epiphysis. J Pediatr Orthop 21: 488-494, 2001.

13. Burton MS: Diagnosis and treatment of adolescent idiopathic scoliosis. Pediatr Ann 42: e233-e237, 2013.

14. Dickson RA and Archer IA: Surgical treatment of late-onset idiopathic thoracic scoliosis. The leeds procedure. J Bone Joint Surg Br 69: 709-714, 1987.

15. Bunnell WP: The natural history of idiopathic scoliosis. Clin Orthop Relat Res: 20-25, 1988

16. Roach JW: Adolescent idiopathic scoliosis. Orthop Clin North Am 30: 353-365, vii-viii, 1999.

17. Miller NH: Cause and natural history of adolescent idiopathic scoliosis. Orthop Clin North Am 30: 343-352, vii, 1999.

18. Tambe AD, Panikkar SJ, Millner PA and Tsirikos AI: Current concepts in the surgical management of adolescent idiopathic scoliosis. Bone Joint J 100-B: 415-424, 2018.

19. Kim HJ, Blanco JS and Wildmann RF: Update on the management of idiopathic scoliosis. Curr Opin Pediatr 21: 55-64, 2009.

20. Chowdhry M, Matsen Ko L, Franklin C and Parvizi J: Reactive scoliosis: A challenging phenomenon in adolescent patients with hip arthritis. Arthroplast Today 3: 160-163, 2017.

This work is licensed under a Creative Commons Attribution-NonCommercial-NoDerivatives 4.0 International (CC BY-NC-ND 4.0) License. 